\title{
Alternativas para classificação de recursos minerais - métodos geoestatísticos tradicionais
}

\author{
(Alternatives for resource classification - \\ traditional geostatistical methods)
}

\section{Resumo}

A dificuldade na quantificação do grau de incerteza associado com a estimativa de recursos minerais tem levado ao desenvolvimento de uma gama de metodologias, termos e definições. Isso gerou uma situação em que praticamente cada companhia de mineração possui sua metodologia particular de classificação. Intuitivamente, sabe-se que os métodos tradicionais utilizados na avaliação de recursos não levam em consideração a continuidade espacial dos teores e, mesmo que algumas metodologias baseadas em métodos geoestatísticos são incapazes de fornecer uma medida do erro associado às estimativas. Nesse sentido, esse artigo se propõe a desenvolver uma metodologia que permita uma análise quantitativa e qualitativa dos recursos minerais estimados por meio da incorporação da incerteza e da adequada definição do risco ou erro associado às estimativas. Um estudo de caso com um banco de dados de minério de ferro típico foi efetuado, permitindo uma comparação, tanto entre os diferentes sistemas de classificação, como entre os parâmetros-chave de cada sistema, sendo que os resultados obtidos permitiram ressaltar as limitações específicas de cada metodologia, além de demonstra a natureza empírica dos métodos tradicionais, devido ao seu caráter predominantemente subjetivo.

\section{Luis Eduardo de Souza}

Dr., Engenheiro de Minas Professor, CSTM/Unipampa E-mail: luissouza@unipampa.edu.br

\section{João Felipe C.L. Costa \\ Dr., Engenheiro de Minas Professor, DEMIN/UFRGS E-mail: jfelipe@ufrgs.br}

\section{Jair Carlos Koppe}

Dr., Engenheiro de Minas/Geólogo
Professor, DEMIN/UFRGS
E-mail: jkoppe@ufrgs.br
Palavras-chave: Estimativa de incerteza, classificação de recursos minerais.

\section{Abstract}

The difficulty in quantifying the degree of uncertainty associated with the estimation of mineral resources has led to the creation of a large suite of methodologies, terms, and definitions, with almost every mining company having its own set of standards. It is intuitively known that traditional methods used to evaluate resources do not take into account the spatial continuity of the grades, and even some approaches based on geostatistical methods are unable to provide a measure of the error associated with their estimates. This article aims at developing a methodology for either quantitative or qualitative analysis of mineral resource estimation through uncertainty incorporation and for the correct definition of the associated risk or error. A comprehensive study about each technique was conducted allowing a comparison among the parameters 
affecting mineral inventory assessment. A case study was conducted with a typical iron ore deposit data set, and the results showed the specific limitations of each classification system and their influence in selecting key parameters. The developed software was used, and the results always demonstrated the empirical nature of the traditional methods based on subjective choices.

Keywords: Uncertainty estimation, mineral resources classification.

\section{Introdução}

O inventário de recursos e reservas minerais é hoje um parâmetro-chave no conjunto de ativos de companhias de mineração, sendo que uma estimativa confiável é crucial, tanto para estudos de viabilidade, quanto para a operação diária de uma mina. A definição das reservas de minério, que tem por base os recursos minerais estimados após a aplicação de uma série de parâmetros técnicos e econômicos, depende de um conjunto de hipóteses e/ou cenários, que, obrigatoriamente, deveriam fazer referência à existência de um nível de erro potencial, alertando-se sobre eles. No entanto, raramente, limites de confiança são claramente definidos e, se eles o são, freqüentemente não levam em consideração muitos dos fatores que provocam incerteza nos teores e nas tonelagens estimadas ou, então, tais limites são baseados em métodos puramente empíricos.

Apesar da crescente preocupação da indústria mineira com o estabelecimento de padrões internacionais de estimativa e de classificação de recursos, nenhum dos principais sistemas de classificação empregados mostra, claramente, como o erro ou nível de confiabilidade associado às estimativas poderia ser calculado (Dowd, 1999; Arik, 1999). A totalidade dos códigos atualmente empregados para classificação de recursos envolve a interação de numerosos critérios qualitativos e quantitativos, tais como a qualidade dos dados e a conti- nuidade geológica e de teores. De-Vitry (2003) discute algumas razões para a não aplicação de critérios ou regras preestabelecidos, apontando, principalmente, para o fato de que, além dessas regras: (i) dificilmente determinadas condições podem ser aplicadas adequadamente para todas as situações ou, mesmo, para diferentes tipos de minérios dentro do mesmo depósito; (ii) que esse fato evitaria que tais regras fossem empregadas indiscriminadamente, sem uma clara compreensão de sua conveniência, acuracidade ou correta aplicação.

Em razão disso, o que se percebe, claramente, é que tanto $(i)$ a falta de normas que prescrevam uma determinada metodologia quanto (ii) a dificuldade na quantificação do erro associado às estimativas fazem com que sejam empregadas, na indústria, de uma maneira geral, as mais variadas abordagens com vistas a tentar determinar essa incerteza, razão pela qual se observa, ainda hoje, o emprego de critérios subjetivos sem embasamento teórico suficiente.

Como nenhum dos atuais códigos de classificação são prescritivos com respeito aos métodos utilizados para estimativas de recursos, ao longo do tempo várias abordagens têm sido sugeridas. Os principais métodos pelos quais os recursos minerais podem ser classificados durante um projeto de avaliação são freqüentemente divididos em dois grupos: os critérios ditos tradicionais ou clássicos (continuidade geológica, densidade amostral, interpolação versus extrapolação, teor de corte, tecnológicos, qualidade dos dados, geométricos, isolinhas, área de influência) e os critérios com abordagem geoestatística (alcance do variograma, variância de krigagem, índice de confiabilidade de recursos, simulação condicional).

Nesse sentido, esse trabalho se propõe a desenvolver uma metodologia de análise quantitativa e qualitativa de recursos minerais que possibilite a incorporação da incerteza às estimativas. Essa metodologia, obrigatoriamente, prevê a implementação computacional das principais técnicas ou métodos empre- gados pela indústria mineira para elaboração de inventários e relatórios de recursos e reservas minerais. O objetivo é permitir um claro e rápido estudo comparativo, em termos de recursos totais, das diversas classes usadas na classificação e do impacto da utilização de cada técnica no inventário final de recursos.

Ilustra-se a metodologia em um estudo de caso com um banco de dados típico de um depósito de minério de ferro. Demonstra-se, que, além de a proposta ser apropriada, para abordar o problema da quantificação da incerteza, possibilitando sua utilização pelos sistemas de classificação de recursos e reservas, ela permite observar, claramente, as limitações de cada técnica e de seus parâmetros-chave. Comprova-se que os resultados variam entre os diversos métodos em função dos critérios adotados por cada usuário, ressaltando, assim, seu caráter subjetivo. Da mesma forma, as ferramentas computacionais desenvolvidas permitiram a realização de uma análise de sensibilidade dos métodos analisados e a confecção de diferentes cenários, além da verificação do impacto nos recursos quando cada um dos diferentes métodos é selecionado.

\section{Metodologia}

Ao longo do tempo, várias abordagens têm sido utilizadas com vistas a categorizar recursos e reservas minerais, sempre tentando responder a algumas questões fundamentais: qual é a quantidade existente do bem mineral, qual o seu teor médio e qual é o grau de certeza associado com essas estimativas?

$\mathrm{Na}$ prática, enquanto as médias e grandes empresas se utilizam de ferramentas modernas de engenharia econômica e geoestatística, já buscando atender às normas internacionais, as pequenas empresas, mesmo perdendo em eficiência empresarial e acuracidade, ainda fazem uso dos métodos tradicionais. No entanto, mesmo os métodos geoestatísticos podem implicar a adoção de critérios subjetivos e ambíguos e, conseqüentemente, estarem sujeitos a diversas contestações. 
Os métodos mais amplamente empregados pelas empresas do setor mineral, para classificação de seus recursos, de maneira geral, são: (a) a densidade amostral na vizinhança de cada bloco a ser estimado, (b) alcance do semivariograma ou distância de correlação e (c) variância de krigagem. $\mathrm{O}$ fato de consistir em uma escolha arbitrária dos parâmetros-chave (por exemplo, a definição do número de amostras necessárias para enquadramento em uma dada categoria ou o percentual do alcance do variograma a ser empregado), impedimentos de caráter prático (a existência de anisotropia zonal impede a utilização de técnicas baseadas no alcance do variograma, pois este só seria atingido no infinito, o que implica em que todos os blocos fossem classificados como recursos medidos) e problemas de embasamento teórico (a variância de krigagem não é uma medida absoluta de erro, já que não considera no seu cálculo os valores dos dados na vizinhança do bloco krigado), por si só, impedem, tanto o emprego irrestrito dos métodos, como sua utilização de maneira padronizada em um dado sistema de classificação.

Nesse sentido, com o objetivo de desenvolver uma metodologia que permitisse a obtenção de uma análise quantitativa e qualitativa dos recursos minerais, incorporando a incerteza às estimativas, entendeu-se que era necessário dispor de uma ferramenta que tornasse possível a obtenção de uma análise de sensibilidade de cada um dos parâmetros utilizados nas técnicas atualmente empregadas, de maneira a aferir o impacto dos mesmos nos inventários de recursos.

Utilizando como plataforma a rotina de interpolação por krigagem $k t 3 d$ do GSLIB - Geostatistical Software Library (Deutsch \& Journel, 1998), foi desenvolvido um programa computacional (MineReC - Mineral Resources Classification). Nesse programa, distintas técnicas de classificação dos recursos (tradicionais e/ou geoestatísticas) podem ser empregadas, permitindo seu enquadramento nas categorias "medido", "indicado" e "inferido".
Desenvolvido em duas linguagens de programação, a interface gráfica do programa foi escrita em Visual Basic, apresentando menus e janelas de fácil utilização, sendo bastante intuitiva. Todas as demais operações foram escritas em linguagem Fortran 90, escolhida devido à sua robustez e estabilidade quando se exigem uma alta performance de processamento e a possibilidade de alocação dinâmica de memória, otimizando o trabalho com grandes matrizes. O programa fornece um avançado sistema de interpolação 3-D para pontos ou blocos, por krigagem simples, ordinária, com modelo de tendência externa e, ainda, por krigagem ordinária convexa, que realiza a correção dos pesos negativos (Deutsch, 1996), exigência para utilização das variâncias como medidas de incerteza e cálculo do erro.

A Figura 1 ilustra a janela-padrão apresentada pelo programa com as opções implementadas para categorização dos recursos minerais baseado em: (i) número de amostras na vizinhança do bloco a ser estimado, opção que pode ser utilizada independentemente ou associada à utilização de uma porcentagem do alcance do variograma ou distância de correlação, (ii) métodos geométricos, com a definição de raios de influência ou por dilatação e erosão, com distâncias de extrapolação definidas pelo usuário, (iii) cálculo do erro da estimativa (David, 1977; Yamamoto, 1999; Souza, 2002), permitindo ao usuário optar entre a utilização do desvio-padrão de krigagem, de interpolação (Froidevaux, 1993; Yamamoto, 1999) ou combinado (Arik, 1999) e do nível de confiança desejado, (iv) ângulo de regressão linear (Mwasinga, 2001).

\section{Discussão de resultados}

Cabe salientar, novamente, que a principal intenção, quando da concepção do programa, não foi fornecer um conjunto de parâmetros, para cada metodologia implementada, que viesse a ser utilizado indistintamente pelo usuário, mas introduzir uma ferramenta que viesse atender a dois aspectos principais: $(i)$ permitisse a realização de uma análise da sensibilidade de cada um dos parâmetros utilizados e (ii) disponibilizasse uma maneira de efetuar um estudo

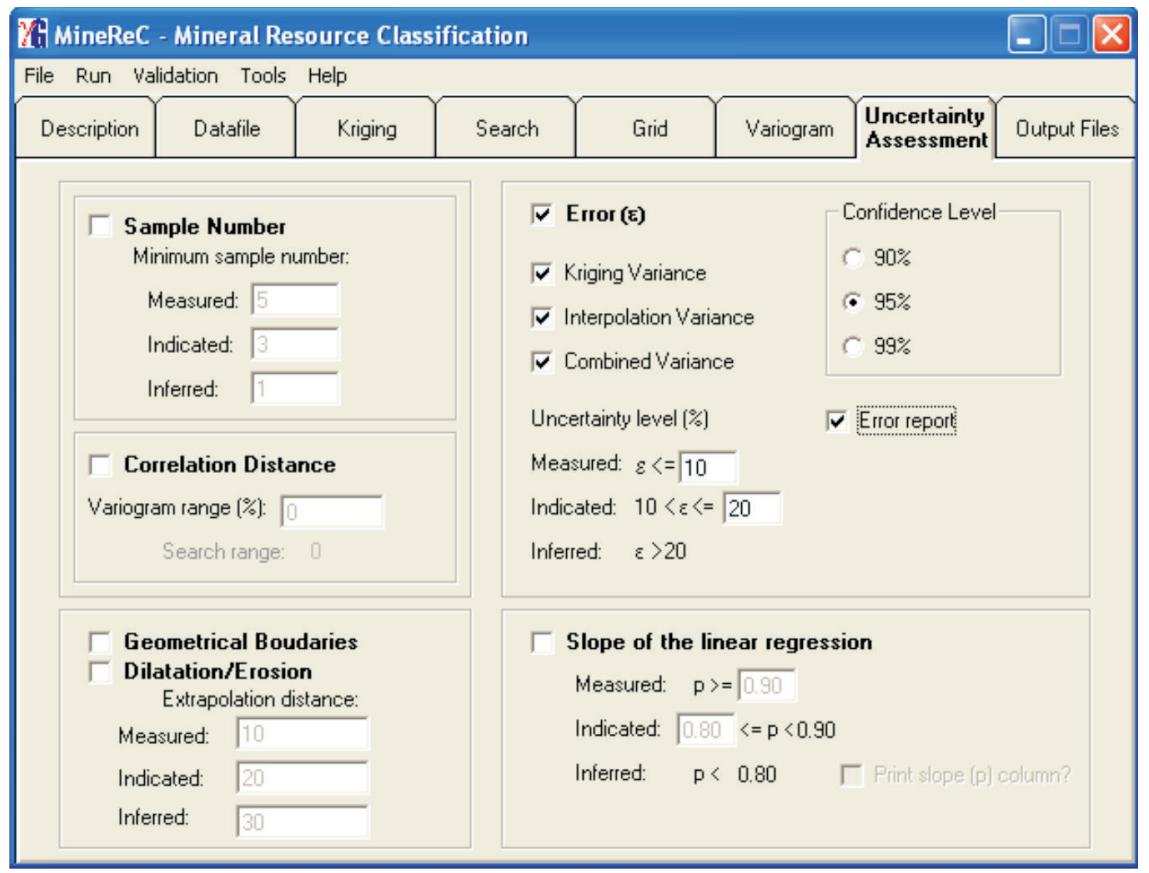

Figura 1 - Janela-padrão para escolha do método e definição dos parâmetros para categorização dos recursos. 
comparativo entre as diferentes metodologias.

Em um arquivo de saída típico, cada bloco recebe um carimbo, correspondente à categoria de recurso, para um dado método de classificação. Com a utilização de uma rotina de classificação, numa base bloco-a-bloco, há uma alta probabilidade de obtenção de blocos de uma determinada categoria cercados por blocos pertencentes a diferentes classes, evidenciando uma textura "salt and pepper" nos mapas de classificação. Dessa forma, foi implementada uma rotina de pós-processamento que visa a corrigir esses problemas, tornando os cenários de classificação mais coerentes e exeqüíveis em termos de lavra. Na metodologia idealizada, o pós-processamento é conduzido comparando-se a classe de um bloco com a percentagem de blocos na sua vizinhança local. Se essa vizinhança apresentar, por exemplo, mais de $80 \%$ dos blocos em uma categoria distinta daquele bloco em análise, o mesmo é reclassificado de acordo com a classe dominante.

Os efeitos da aplicação desse procedimento de pós-processamento foram, também, analisados e, para este estudo de caso, a quantidade de blocos reclassificados foi considerada irrelevante em relação ao cenário total de recursos, sendo que menos de $1 \%$ dos blocos originalmente classificados como "medidos" foram alterados.

\section{Número de amostras}

Nesse método, os blocos são classificados em função de um número mínimo de amostras encontradas em um volume de busca. Geralmente, o volume de busca é definido como uma percentagem do alcance do variograma, uma vez que esses alcances indicam as distâncias onde os dados exibem correlação espacial.

$\mathrm{Na}$ Figura 2, são ilustrados os resultados em termos de número de blocos classificados em cada categoria de recurso (medido, indicado e inferido).
Quando foi empregado o método de classificação, apenas o número de amostras utilizadas foi estimado para cada bloco. Esse mesmo critério foi aplicado em conjunto com uma redução da vizinhança de busca, ou seja, para ser classificado em uma determinada classe, o bloco precisa de ter aquele número de amostras definidas pelo usuário em até $70 \%$ da distância de alcance do variograma. No eixo horizontal, são apresentados os números mínimos de amostras para incluir um bloco em cada classe, isto é, $3 \times 2 \times 1$ significa ao menos 3 amostras classificado como medido, ao menos $2 \mathrm{o}$ bloco é classificado como para indicado e ao menos uma o bloco é classificado como inferido. Como o número mínimo de amostras, para a categoria de recursos, aumenta, a quantidade de blocos satisfazendo as restrições reduz, fazendo com que os blocos migrem de categorias. Isto explica a redução de recursos medidos a o incremento de indicados e inferidos com o aumento do número mínimo de amostras.

O exemplo corrobora o que já era esperado, ou seja, com a imposição de um incremento no número mínimo de amostras necessárias, há um decréscimo de blocos classificados nas categorias de recursos e um aumento de blocos não classificados, por não atenderem

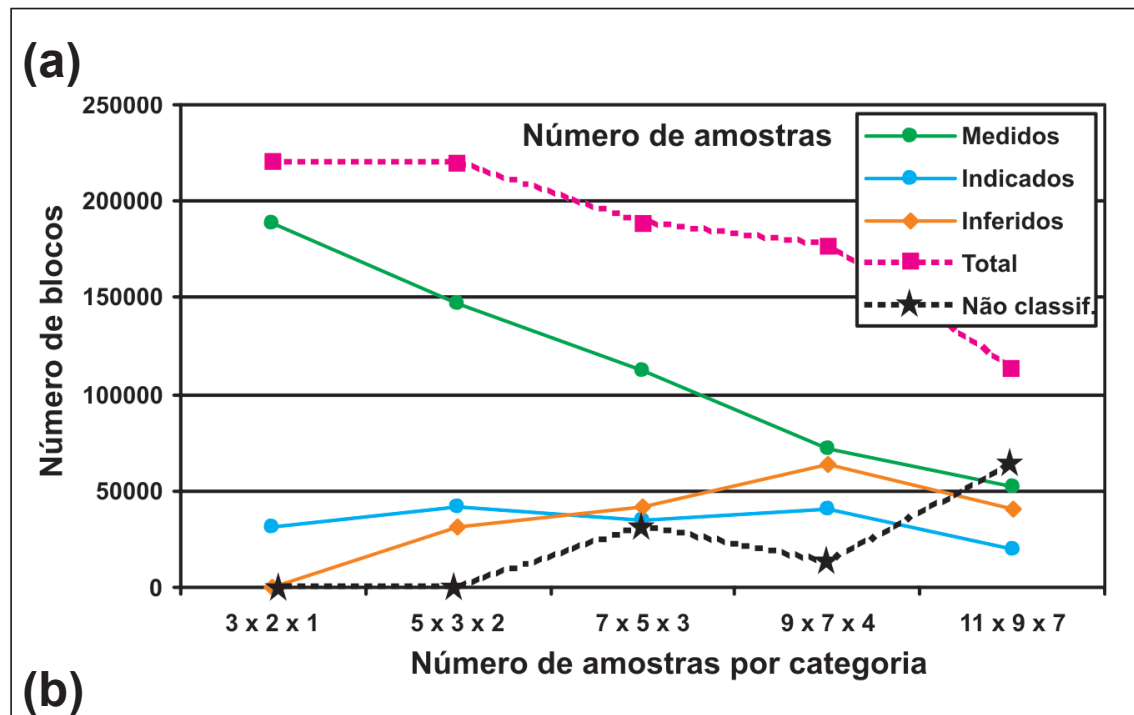

(b)

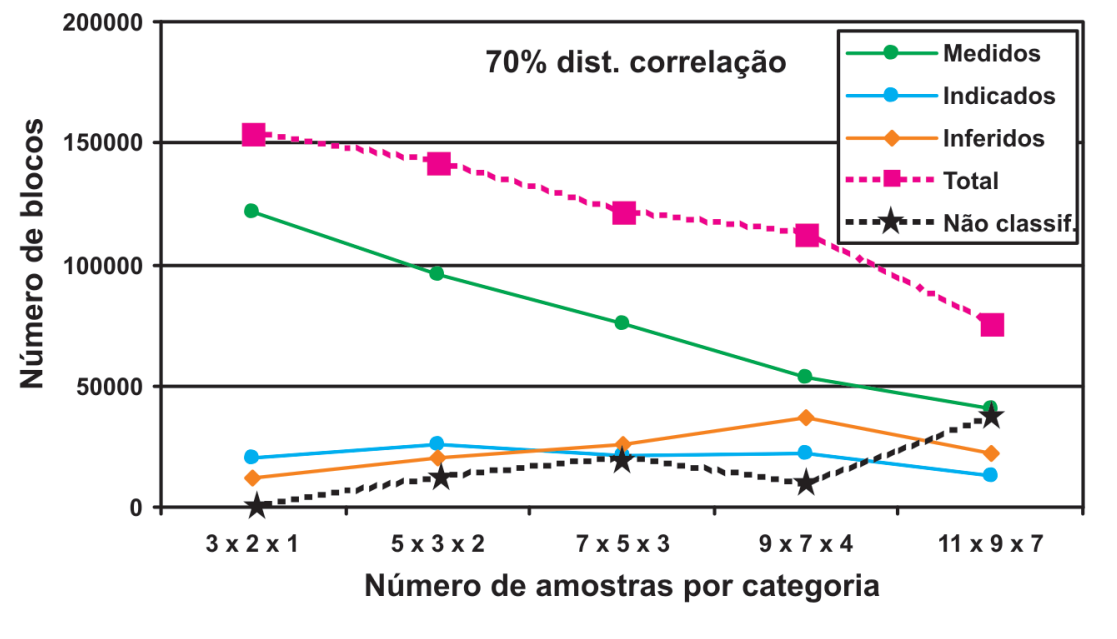

Figura 2 - Comparação em termos de classificação de recursos utilizando o critério baseado apenas (a) no número mínimo de amostras para estimar um dado bloco e (b) no número mínimo de amostras em uma distância máxima de $70 \%$ do alcance do variograma. 
aos critérios estabelecidos. Intuitivamente, sabe-se que, quanto mais rigoroso o critério, demandando um número maior de amostras, menor será o erro associado com as estimativas. No entanto, não há condições de se saber qual o nível de erro praticado. Da mesma forma, um excesso de rigorismo para obter um erro baixo certamente pode ser perigoso, na medida em que acarretará uma perda acentuada de recursos.

\section{Erro-padrão da estimativa}

A krigagem ordinária $(\mathrm{KO})$ é, provavelmente, a técnica geoestatística mais amplamente empregada para estimativas de recursos minerais, tanto devido ao seu caráter robusto, quanto às suas características relacionadas com a quantificação da incerteza pela variância de krigagem (Matheron, 1963; David, 1977; Journel, 1983; Isaaks \& Srivastava, 1989). No entanto, a variância de krigagem sempre recebeu várias críticas pelo fato de não reconhecer a variabilidade local dos dados, um importante aspecto quando depósitos minerais heterogêneos com áreas de altos e baixos valores estão sendo avaliados. Como a variância obtida pela krigagem ordinária mede apenas a configuração espacial dos dados vizinhos, essa medida para incerteza não deveria ser utilizada para classificar blocos estimados. Assim, alternativas à variância de krigagem têm sido propostas e, nesse trabalho, duas abordagens distintas, para o cálculo da variância da estimativa, a partir dos pesos da krigagem ordinária, são utilizadas: (i) a variância de interpolação (Yamamoto, 1999) e (ii) a variância combinada (Arik, 1999).

Assim, pode-se calcular o erro-padrão com base em cada uma das variâncias alternativas, assumindo-se um intervalo de confiança de $95 \%$ e uma distribuição Gaussiana dos erros (David, 1977). Na Figura 3, os blocos foram categorizados em função das faixas de erro estabelecidas: entre 0 e $5 \%$ o bloco foi classificado como medido, entre 5 e $10 \%$ o bloco foi classificado como indicado e maior que $10 \%$, o bloco foi classificado como inferido. (a)

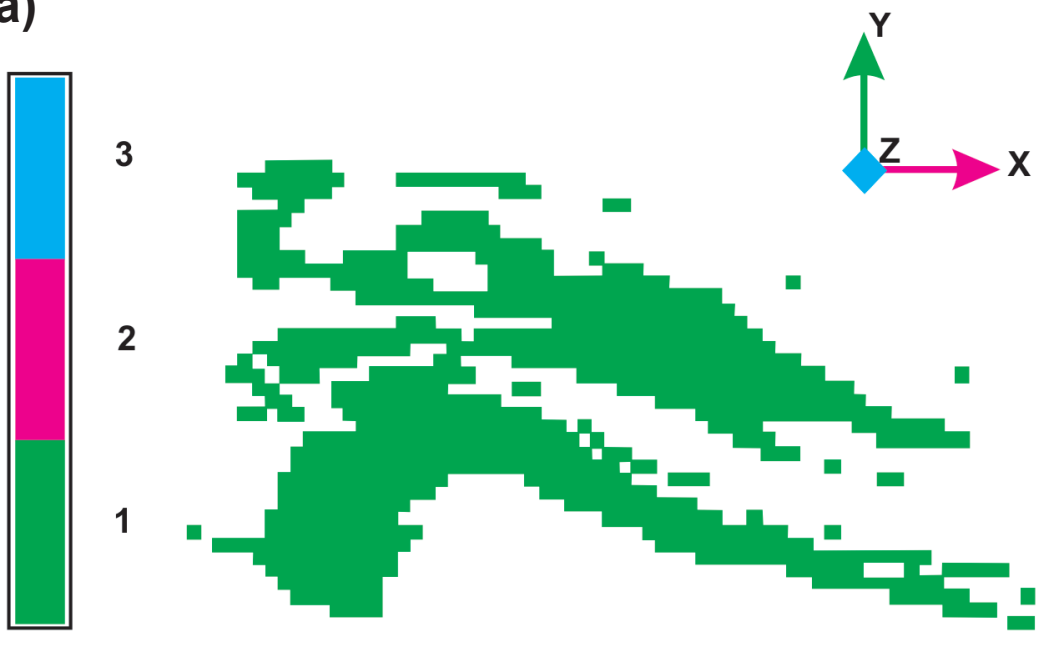

(b)

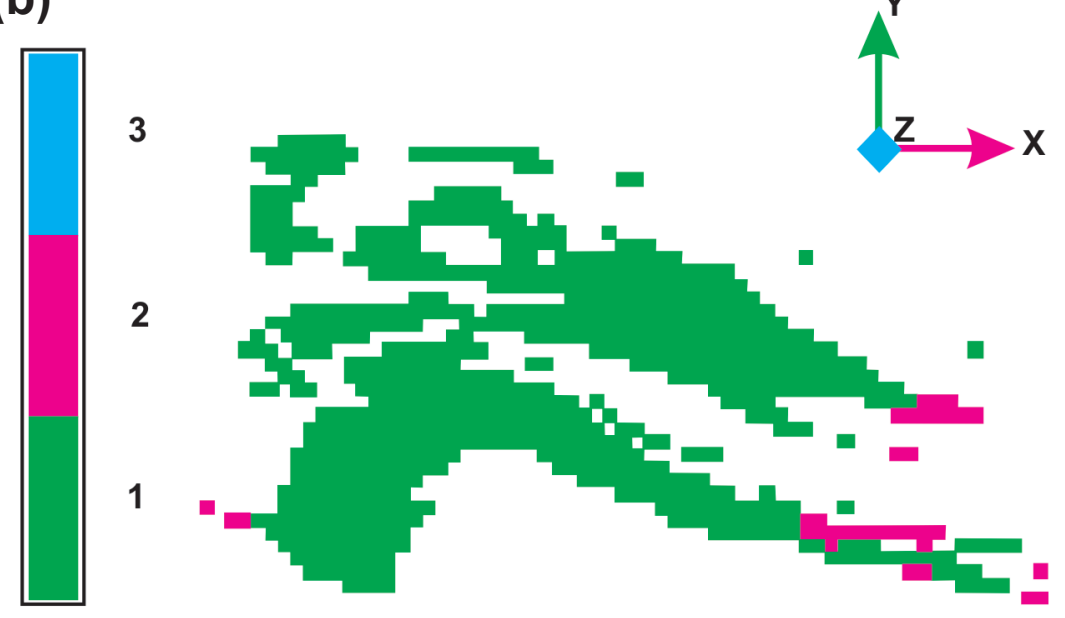

(c)

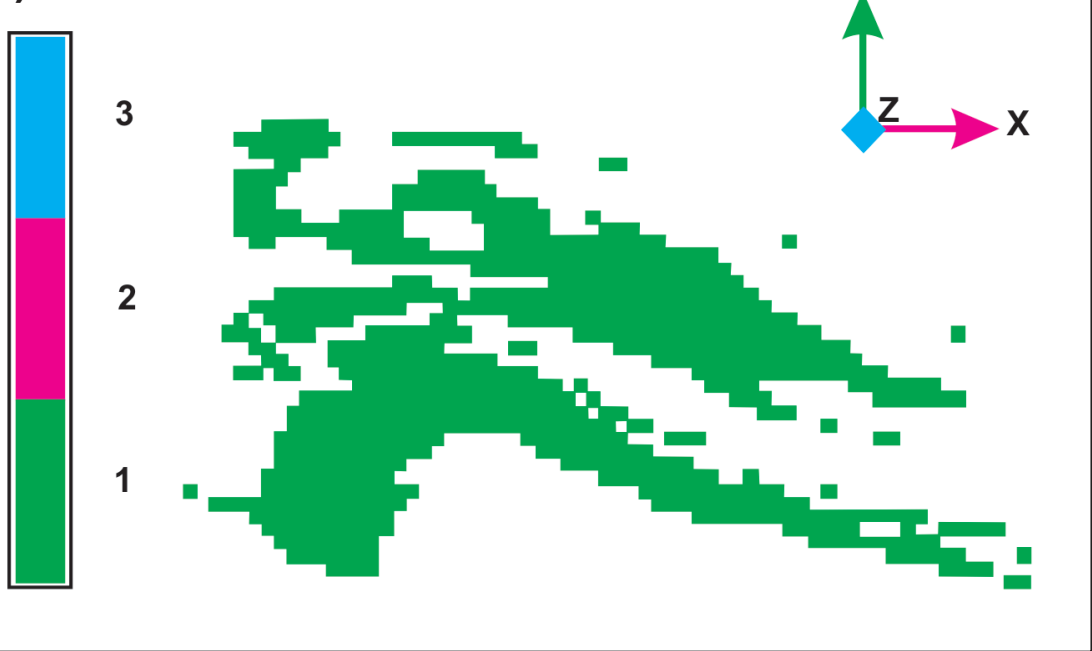

Figura 3 - Categorias de recursos para o nível 970 m, baseado no erro-padrão calculado a partir da (a) variância de krigagem, (b) variância de interpolação e (c) variância combinada, sendo 1: blocos medidos, 2: indicados e 3: inferidos. 
Percebe-se que cada tipo de variância leva a diferentes cenários de variabilidade, que reflete nos erros calculados e, portanto, na categoria dos blocos. Como, nesse estudo de caso, a variável analisada apresentava uma baixa variabilidade dentro do domínio geológico estudado, as mudanças na variabilidade local não afetaram, significativamente, $\mathrm{o}$ número de blocos em cada categoria com a mudança de abordagem.

\section{Ângulo de regressão linear}

O ângulo de regressão linear foi, originalmente, concebido como um teste para avaliar a qualidade das estimativas e consiste no cálculo dos principais parâmetros da regressão entre os valores estimados $\left(Z_{v}^{*}\right)$ e reais $\left(Z_{v}\right)$ para cada bloco, assumindo-se as hipóteses de que (i) o variograma seja válido e que (ii) a regressão seja linear. Como os valores reais são desconhecidos, o ângulo de regressão linear " $\rho$ " é obtido utilizandose as covariâncias entre as amostras utilizadas para estimar um dado bloco e aquele bloco, sendo que as estimativas seriam mais próximas da realidade (menor o erro) quanto mais próximo de 1 fosse $o$ valor de $\rho$.

Como foi previamente ressaltado, devido à baixa variabilidade dos teores de minério de ferro utilizados nesse estudo de caso e à substancial quantidade de dados disponíveis no depósito, a maioria dos valores calculados de $\rho$ ficaram próximos de 1 . A Tabela 1 apresenta o número de blocos classificados em cada intervalo de valores de $\rho$. O esquema de classificação adotado define como recurso medido os blocos onde $\rho \geq \rho_{1}$, como recurso indicado os blocos do intervalo $\rho_{1}>\rho \geq \rho_{2}$ e, como inferidos, os blocos com $\rho<\rho_{2}$.

De acordo com a Tabela 1 , há uma variação no número de blocos em cada categoria de $\rho$ adotado, com um aumento de blocos nas categorias de recursos indicado e inferido e um decréscimo de recursos medidos com o incremento de $\rho$. O método leva a resultados lógicos de que apenas blocos com alto grau de reconciliação (ângulo de regressão próximo de 1) são elegíveis para a categoria de recursos medidos.

\section{Limites geométricos ou área de influência}

Os métodos incluídos nesse grupo são ainda largamente utilizados na indústria mineira, justificando sua análise e implementação. Duas variantes foram investigadas: ( $i$ ) área de influência e (ii) dilatação-erosão. No entanto, na medida em que esses métodos não fornecem uma medida direta da incerteza, como sugerido pelos principais códigos de classificação, há um tendência em se evitar sua utilização.

No primeiro método, adota-se uma área de influência subjetiva (distância) em torno dos furos de sondagem como critério para discriminação entre recursos. De acordo com a distribuição espacial dos dados (distância média entre os dados amostrais disponíveis e distância máxima de extrapolação), tenta-se interpretar, empiricamente, a continuidade geológica. Já o método de dilatação-erosão, bastante empregado em minas de ferro no Brasil (Guimarães et. al, 2004) seria um tipo de análise de morfologia matemática capaz de distinguir entre zonas de interpolação e extrapolação, assumindo, com base em evidência geológicas, que uma determinada malha de sondagem (geralmente $100 \times 100$ metros) é apropriada para delinear com alto grau de confiança os corpos de minério de ferro.
Na Figura 4, é apresentada uma seção horizontal das categorias de recursos no nível $970 \mathrm{~m}$, definidas em função das áreas de influência. As distâncias selecionadas na definição das classes ao redor de cada furo de sonda foram de $75 \mathrm{~m}$ para medido, 125 para indicado e 150 para inferido.

\section{Análise combinatória dos métodos de classificação}

Conforme apresentado nas seções anteriores, cada uma das metodologias analisadas categoriza os blocos em classes de recursos utilizando diferentes abordagens, cada uma, no entanto, tentando acessar a incerteza associada com as estimativas. Percebe-se que, enquanto os métodos baseados em abordagens geoestatística tentam utilizar uma medida numérica para a incerteza, os métodos tradicionais utilizam uma incerteza subjetiva normalmente associada com a densidade amostral.

A partir de cada cenário de recursos individual, obtido pelas metodologias testadas, optou-se por verificar como cada bloco seria classificado utilizando diversos métodos de classificação diferentes simultaneamente, ou, mesmo, alterando os parâmetros-chave que controlam cada método. $\mathrm{O}$ método

Tabela 1 - Número de blocos classificados em cada intervalo de valores de $\rho$.

\begin{tabular}{c|c|c|c|c}
\hline \multicolumn{2}{c|}{ Ângulo de Regressão } & \multicolumn{3}{|c}{ Recursos } \\
\hline$\rho_{1}$ & $\rho_{\mathbf{2}}$ & Medidos & Indicados & Inferidos \\
\hline 0.95 & 0.90 & 20573 & 1269 & 2429 \\
\hline 0.95 & 0.80 & 20573 & 2392 & 1306 \\
\hline 0.90 & 0.80 & 21842 & 1123 & 1306 \\
\hline 0.90 & 0.70 & 21842 & 1655 & 774 \\
\hline 0.80 & 0.70 & 22965 & 532 & 774 \\
\hline 0.80 & 0.60 & 22965 & 977 & 329 \\
\hline 0.70 & 0.60 & 23497 & 445 & 329 \\
\hline 0.70 & 0.50 & 23497 & 774 & 0 \\
\hline
\end{tabular}


consiste em verificar a freqüência que um dado bloco tem em pertencer a uma categoria de recursos. Assim, um bloco com uma alta probabilidade de pertencer a uma dada categoria, mesmo após a aplicação de múltiplos métodos e/ou com variações internas de seus parâmetros de controle, garantiria uma razoável robustez em sua categorização.

Para ilustrar essa metodologia, 32 cenários foram testados, envolvendo diferentes métodos de categorização de recursos e reservas e várias escolhas de parâmetros de cada método. Escolhido um bloco ao acaso para exemplo, constata-se que o mesmo possui uma probabilidade de $84 \%$ ( 27 de 32 possibilidades) de ser atribuído à categoria de recursos medidos. Assim, independentemente do tipo de abordagem escolhida como critério de classificação, esse bloco, provavelmente, acabaria sendo classificado como recurso medido.

Em se extendendo o procedimento para todos os blocos do modelo, é possível obter-se, então, um modelo de blocos com a distribuição de probabilidades dos blocos pertenceram à categoria de recurso medido, contemplando-se o conjunto de 32 cenários testados

\section{Conclusões}

O inventário de recursos e reservas minerais é um parâmetro-chave no conjunto de ativos de companhias de mineração, já que sua estimativa confiável é crucial, tanto para estudos de viabilidade, quanto para a operação diária de uma mina. A definição das reservas de minério, que tem por base os recursos minerais estimados após a aplicação de uma série de parâmetros técnicos e econômicos, depende de um conjunto de hipóteses e/ou cenários, que, obrigatoriamente, deveriam fazer referência à existência de um nível de erro potencial, alertando-se sobre eles.

Esse estudo compara diferentes abordagens utilizadas para quantificação e classificação de recursos, ilustrando

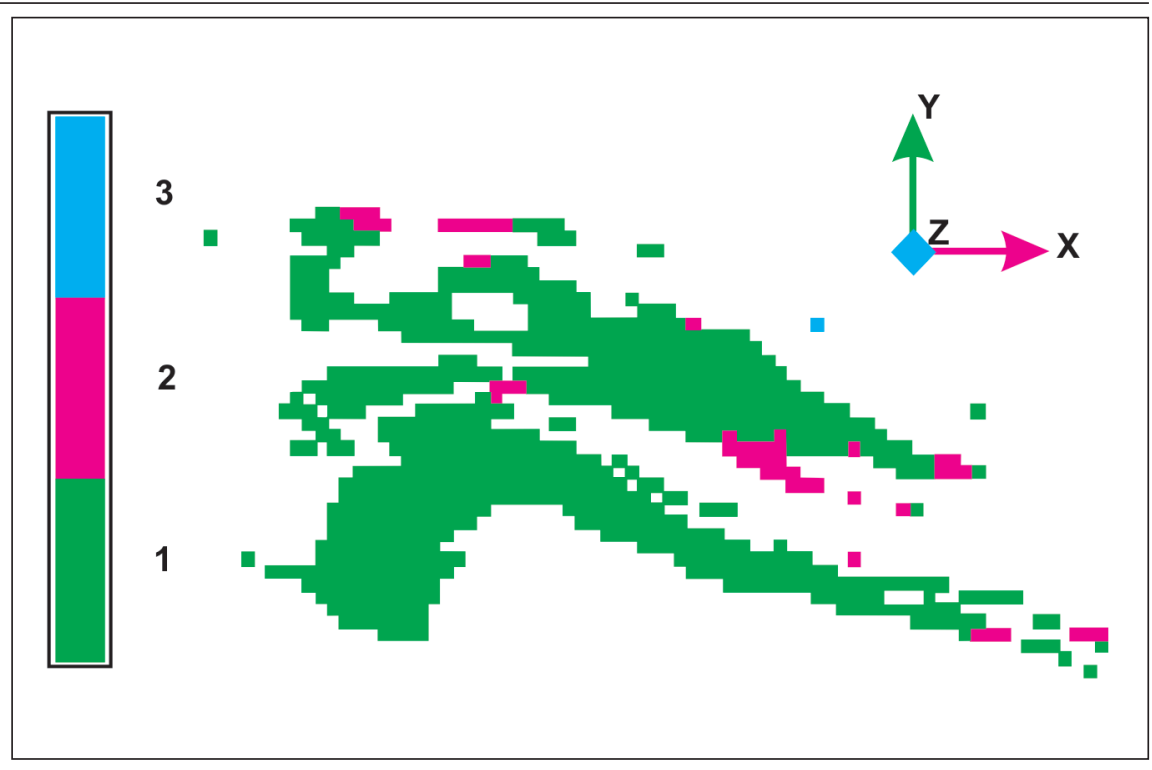

Figura 4 - Seção horizontal do nível 970 m das categorias de recursos em função das áreas de influência, sendo 1: medido, 2: indicado e 3: inferido.

sua aplicação num depósito típico de minério de ferro, sendo que a maioria das metodologias é utilizada rotineiramente pela indústria, justificando uma análise dos resultados que cada método disponibiliza. Todas as metodologias abordadas foram implementadas computacionalmente utilizando o MineReC e foram mostrados o impacto que cada parâmetro de controle tem sobre os métodos específicos e o impacto em se optar por um ou outro método.

Os métodos de densidade amostral, apresentados aqui, têm um aspecto bastante curioso no que diz respeito à própria percepção da diferença entre erro e incerteza. Intuitivamente, sabe-se que, quanto mais forem aumentadas as exigências em termos de número de amostras, para classificar um determinado bloco, menor será o erro associado à estimativa, no entanto continua-se sem nenhuma informação em qual patamar este erro estará flutuando. Da mesma forma, um excesso de rigorismo com o intuito de se obter um erro baixo certamente será perigoso, podendo, inclusive, inviabilizar uma operação com a perda excessiva de recursos minerais, conforme observado nos resultados obtidos.

Salientaram-se, nesse trabalho, os problemas de embasamento teórico rela- cionados com a utilização da variância de krigagem como uma medida de incerteza e foram apresentadas duas alternativas que se propõem corrigir essas deficiências. Os resultados apresentados mostram que pode haver um impacto considerável no inventário de recursos quando cada uma das opções é utilizada.

Entende-se, que, tanto os métodos geométricos aqui apresentados, como o de ângulo de regressão linear, não fornecem, propriamente, medidas de incerteza, mas fornecem muito mais indicativos da distribuição espacial das amostras utilizadas para krigar um dado bloco. Dessa forma, tais métodos não atendem as últimas recomendações dos códigos internacionais para classificação de recursos e reservas.

Como salientado anteriormente, não foi objetivo desse trabalho apontar a melhor ou pior técnica, mas por meio dos resultados obtidos pode-se destacar dois aspectos principais: $(i)$ o caráter extremamente subjetivo da aplicação de determinadas metodologias, na medida em que pequenas alterações nos parâmetros podem impactar, significativamente, inventário de recursos e (ii) a importância de serem realizados testes e análises de sensibilidade, tanto para definição dos parâmetros, quanto para atestar os re- 
sultados obtidos por diferentes métodos de classificação.

\section{Agradecimentos}

Os autores gostariam de agradecer ao CNPq e à Vale - Companhia Vale do Rio Doce pela cooperação e suporte para realização deste estudo.

\section{Referências \\ bibliográficas}

ARIK, A. An alternative approach to resource classification. In: INTERNATIONAL SYMPOSIUM ON COMPUTER APPLICATIONS IN THE MINERAL INDUSTRIES (APCOM'99), 28. Proceedings... USA: Colorado School of Mines, Golden, 1999. p. 45-53.

DAVID, M. Geostatistical ore reserve estimation. Developments in Geomathematics 2. Amsterdam: Elsevier Scientific Publishing Company, 1977. $364 \mathrm{p}$.

DEUTSCH, C.V., JOURNEL, A.G. GSLIB: Geostatistical Software Library and
User's Guide. (2. ed.). New York: Oxford University Press, 1998. 369 p. 1998.

DEUTSCH, C.V. Correcting for negative weights in ordinary kriging. Computers \& Geosciences, v. 22, n.7, p. 765-773, 1996.

DE-VITRY, C. Resource classification - a case study from the Joffre-Hosted iron ore of BHP Billiton's Mount Whaleback operations. Mining Technology, Trans. Inst. Min. Metall., v. 112, p. A185-A196, 2003.

DOWD, P.A. Reserves and resources - continuity and levels of confidence. In: INTERNATIONAL SYMPOSIUM ON COMPUTER APPLICATIONS IN THE MINERAL INDUSTRIES (APCOM'99), 28. Proceedings... Colorado, USA: Colorado School of Mines, Golden, 1999. p.93-99.

FROIDEVAUX, R. Constrained kriging as an estimator of local distribution functions. CAPASSO, V., GIRONE, G., POSA, D. (ed.). In: INTERNATIONAL WORKSHOP ON STATISTICS OF SPATIAL PROCESSES: THEORY AND APPLICATIONS. Proceedings... Bari, Italy, p. 106-118, 1993.

ISAAKS, E.H., SRIVASTAVA, M.R. An introduction to applied geostatistics. New York: Oxford University Press, 1989. 561 p.

JOURNEL, A.G. Non-parametric estimation of spatial distributions. Mathematical Geology, V. 15 , n. 3 , p. $445-468,1983$

MATHERON, G. Principles of geostatistics. Economic Geology, n. 58, p. 1246-1266, 1963.

MWASINGA, P.P. Approaching resource classification: general practices and the integration. In: INTERNATIONAL SYMPOSIUM ON COMPUTER APPLICATIONS IN THE MINERAL INDUSTRIES (APCOM'2001), 29. Proceedings... Beijing, China, 2001. p. $97-104$.

SOUZA, L.E. Estimativa de incertezas e sua aplicação na classificação de recursos minerais. Universidade Federal do Rio Grande do Sul: Programa de Pós-Graduação em Engenharia de Minas, Metalúrgica e de Materiais (PPGEM), 2002. 171 p. (Dissertação de Mestrado).

YAMAMOTO, J.K. Quantification of uncertainty in ore-reserve estimation: applications to chapada copper deposit, state of Goiás, Brazil. Natural Resources Research, v. 8, n. 2, p. 153-163, 1999.

Artigo recebido em 11/11/2008 e aprovado em 21/05/2009.

\section{A REM tem novo endereço: FUNDAÇÃO GORCEIX - REM Rua Carlos Walter Marinho Campos, 57 Bairro: Vila Itacolomy 35400-000 - Ouro Preto - MG $\begin{array}{ll}\text { (31) } 3551-4730 & \text { (31) } 3559-7408\end{array}$ www.rem.com.br}

